\title{
Reply to letter to the editor: Subfoveal Choroidal Thickness as a Predictor of Treatment Response to Anti-Vascular Endothelial Growth Factor Therapy for Polypoidal Choroidal Vasculopathy
}

\author{
Hyesun Kim ${ }^{1,2}$ - Sung Chul Lee ${ }^{1} \cdot$ Kye Yoon Kwon ${ }^{1}$. Christopher Seungkyu Lee ${ }^{1}$
}

Received: 15 April 2016 / Accepted: 19 April 2016/Published online: 26 April 2016

(C) Springer-Verlag Berlin Heidelberg 2016

\section{Dear Editor,}

We thank Dr. Uzun et al. for their interest in our paper. Most of their concerns could perhaps be explained in terms of inherent limitation of retrospective study. We did not analyze axial length in our patients due to limited data availability. However, we found no significant difference in refractive error between good-responders and poor-responders $(+0.41 \pm 1.55$ diopter vs. $-0.25 \pm 1.24$ diopter, $P=0.157)$. Since axial length and refractive error are highly correlated $[1,2]$, we may safely assume that there would be no significant difference in axial length between the two groups. Therefore, we do not think that a lack of axial length analysis should affect our finding.

We evaluated the baseline choroidal thickness as the predictor of treatment response. Thus, the possibly raised intraocular pressure from intravitreal injections and their effects on choroidal thickness should not be a relevant issue in our study. There was no difference in baseline

Christopher Seungkyu Lee

sklee219@yuhs.ac

1 The Institute of Vision Research, Department of Ophthalmology, Yonsei University College of Medicine, 134 Shinchon-dong, Seodaemun-gu, Seoul 120-752, Korea

2 Siloam Eye Hospital, Seoul, Korea intraocular pressure between the two groups (data not shown).

The diurnal variation and other local or systemic conditions that may affect the choroidal thickness and the use of two different drugs (ranibizumab and bevacizumab) are a possible bias in our retrospective study, as discussed in the manuscript. The subgroup analysis of 47 patients who received ranibizumab only confirmed the significantly thinner choroidal thickness in good responders $(225 \pm 94 \mu \mathrm{m}$ vs. $284 \pm 125 \mu \mathrm{m}$, $P=0.037$ ). Further prospective studies that include analyses suggested by Uzun et al. are warranted to validate our findings.

\section{References}

1. Meng W, Butterworth J, Malecaze F, Calvas P (2011) Axial Length of Myopia: A Review of Current Research. Ophthalmologica 225(3):127-134

2. Mallen EA, Gammoh Y, Al-Bdour M, Sayegh FN (2005) Refractive error and ocular biometry in Jordanian adults. Ophthalmic Physiol Opt 25(4):302-309 